\title{
Democracia, participación y ciudadanía europea: recursos didácticos cartográficos para la enseñanza de la convivencia y la integración europea
}

Democracy, participation and European citizenship: mapping instructional resources for teaching European coexistence and integration

Rafael de Miguel González*, María Sebastián-López*

\section{Resumen}

La enseñanza de las confrontaciones (políticas, étnicas, raciales, religiosas, etc.) producidas a lo largo del siglo XX, y en especial durante la Segunda Guerra Mundial sigue siendo un tema controvertido en la didáctica de las Ciencias Sociales. Tomando como referencia el currículo de cuarto de la ESO, y en especial el bloque décimo de la materia de Geografía e Historia, esta comunicación selecciona una serie de materiales didácticos cartográficos de indudable utilidad en los procesos de enseñanza-aprendizaje de la relación entre el pasado, el presente y la proyección al futuro a través de estas dos disciplinas referentes. Además del estándar de aprendizaje referido al Holocausto, esta propuesta didáctica -fundamentada en los proyectos europeos Youthmetre y European Values Study- procura dar cumplimiento a la comprensión de las causas por las que una Europa en guerra durante el siglo XX ha podido llegar a una unión económica y política en el siglo XXI.

Palabras clave: Ciudadanía europea. Ciudadanía espacial. Participación. Democracia.

\begin{abstract}
Teaching confrontations (political, ethnic, racial, religious, etc.) carried out throughout the 20th century, and especially during the Second World War, is still a controversial issue in Social Sciences Education. Following the curriculum for the fourth year of secondary education, for the subject of Geography and History, this paper selects a series of useful cartographic instructional materials for the teaching-learning processes of the relationship between the past, the present and the projection into the future through these two disciplines. In addition to the learning standard referring to the Holocaust, this didactic proposal -based on the European Youthmetre and European Values Study projects- seeks to fulfill the understanding of the causes why Europe at war during the 20th century has been able to reach a union economic and political in the XXI century.
\end{abstract}

Key words: European citizenship. Spatial citizenship. Participation. Democracy.

\footnotetext{
* Universidad de Zaragoza, Grupo de investigación ARGOS, IUCA • R. de Miguel (ORCID 0000-00029347-5296). M. Sebastián (ORCID 0000-0002-7646-0826) • Autora de correspondencia: María Sebastián (msebas@unizar.es).
}

De Miguel, R. y Sebastián, M. (2020). Democracia, participación y ciudadanía europea: recursos didácticos cartográficos para la enseñanza de la convivencia y la integración europea. CLIO. History and History teaching, 46, 14-29. https://doi.org/10.26754/ojs clio/clio.2020465256. Recibido: 9/9/2020. Aceptado: 24/11/2020. 


\section{Ciudadanía europea: un debate escolar tardío e inacabado.}

La ciudadanía europea es un concepto jurídico que se crea en el Tratado de la Unión Europea, en su versión inicial del Tratado de Maastricht (1992), y que perdura en sus revisiones posteriores del Tratado de Amsterdam (1996), Niza (2000) y Lisboa (2007), así como en el vigente texto consolidado publicado en 2012. En su artículo 9 se indica que "la ciudadanía de la Unión se añade a la ciudadanía nacional sin sustituirla", estableciendo así un valor de complementariedad frente al de exclusión o de confrontación entre naciones que dominó el escenario europeo desde finales del siglo XIX y durante la primera mitad del siglo XX, y que desembocó en las dos guerras mundiales.

A su vez, el Tratado de Funcionamiento de la Unión Europea (aprobado también en Lisboa en 2007), en su vigente texto consolidado (igualmente de 2012), desarrolla los principios de la ciudadanía, entre los que se encuentra la "no discriminación por motivos de sexo, de origen racial o étnico, religión o convicciones", los derechos básicos de los ciudadanos europeos, especialmente, circular y residir libremente en el territorio de los Estados miembros y, en consecuencia, a estudiar y trabajar en el territorio de los Estados miembros. La ciudadanía europea se fundamenta en la protección de los derechos humanos y las libertades fundamentales, conforme al Convenio Europeo del Consejo de Europa, ratificado en 1950, esto es, dos años después de la Declaración Universal de los Derechos Humanos de la Organización de Naciones Unidas e inspirado directamente en ella por medio de principios como el derecho a la vida, la prohibición de la tortura, la esclavitud o el trabajo forzado, el derecho a la libertad física, a la seguridad a un proceso equitativo, así como a las libertades individuales.

Esto es, el proceso de construcción europea se ha ido asentando desde sus inicios hasta la actualidad en los valores liberales, democráticos y del estado de derecho opuestos a los del contexto de los regímenes autoritarios y totalitarios que impulsaron y justificaron procesos como el del Holocausto. Sin embargo, la consideración de la importancia de la educación en los procesos de adquisición de la ciudadanía europea ha sido reciente, resulta inacabada, y sobre todo no siempre aparece diferenciada de otras escalas de ciudadanía: cuando se debate sobre educación para la ciudadanía, se suele mezclar los conceptos de educación para la ciudadanía global, la educación 
para la ciudadanía europea y la educación para la ciudadanía democrática, esencialmente referida a la ciudadanía nacional a que se refería el tratado de la Unión Europea.

Sin embargo, la educación para la ciudadanía es un concepto esencialmente democrático y europeo, ya que en Estados Unidos se habla más de educación cívica (civic education) dentro de la asignatura de los Social Studies en educación secundaria que prepara para una asignatura en bachillerato del sistema Advanced Placement denominada "Política y Gobierno de los Estados Unidos". En Iberoamérica, la educación ciudadana no tiene el mismo grado de desarrollo y consolidación que en Europa por la propia historia de los países al sur del río Bravo, tal y como se ha señalado recientemente en sendos homenajes a dos referentes españoles de la educación para la ciudadanía en nuestro área de Didáctica de las Ciencias Sociales, los recordados profesores Joan Pagés y Ernesto Gómez (Jara y Santisteban, 2018; Pagés y García, 2020). En otras regiones del mundo como el continente africano, Oriente Medio, el Sudeste Asiático o el Pacífico la educación para la ciudadanía suele identificarse especialmente con una educación patriótica (Lee et al., 2004) debido a la propia naturaleza no democrática que sigue rigiendo en la mayoría de los países de estas áreas, tal y como se comprueba en la figura 1.

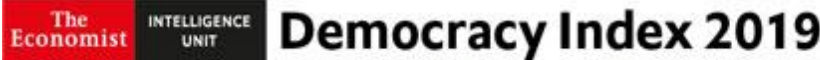

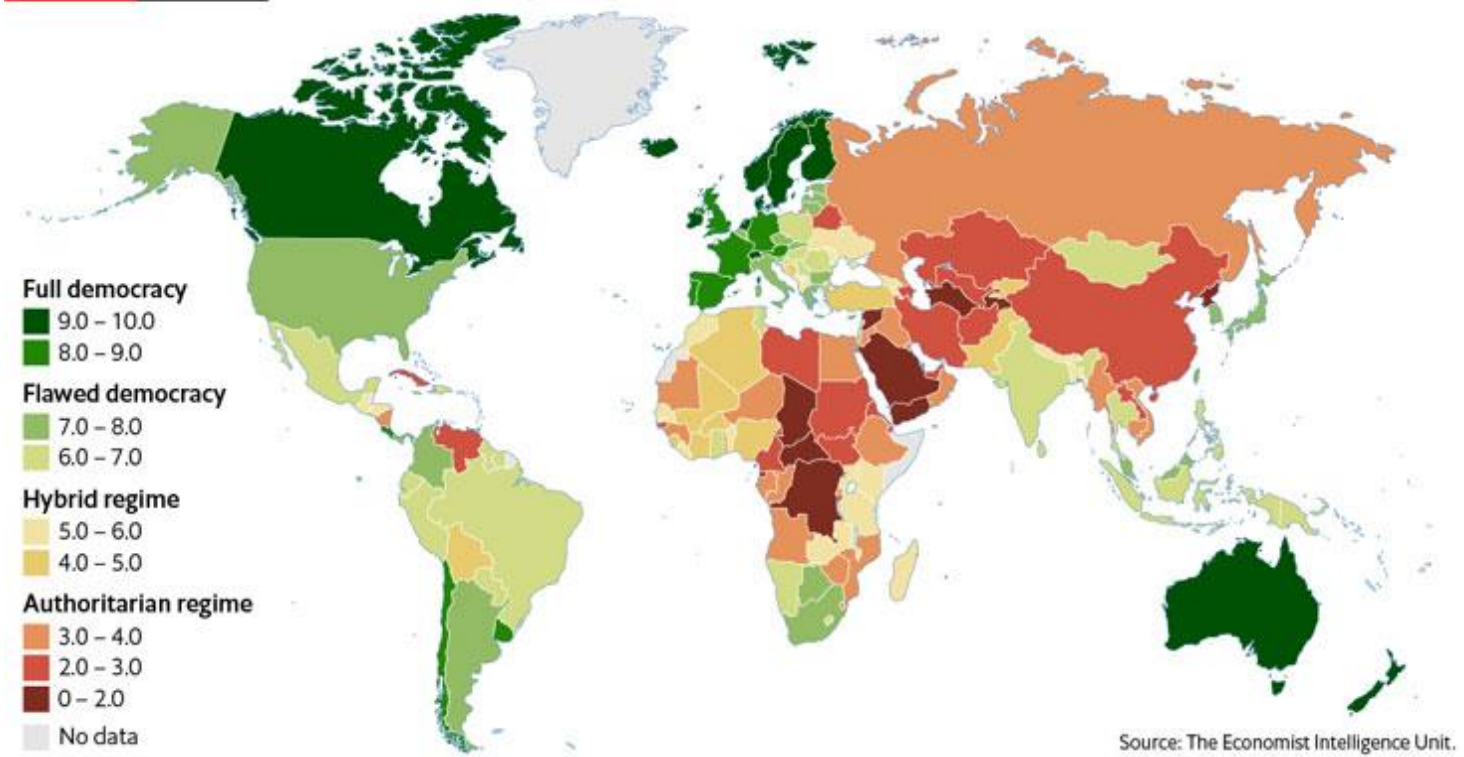

Figura 1. Mapa del índice de democracia (Economist Intelligence Unit. World Democracy report, 2019). 
La educación para la ciudadanía es también un concepto esencialmente europeo, debido a que las instituciones europeas has elaborado diferentes documentos de referencia. Sin entrar en el detalle y contenido de ellos, así como su aplicación por los diferentes Estados miembros de la Unión Europea, que ha sido ya expuesto y analizado en diversas publicaciones (Caballero et al, 2016; Comisión Europea / Eurydice, 2017; Massip y Santisteban, 2020), merece la pena recordar la Recomendación del Consejo de Ministros del Consejo de Europa a los cuarenta y siete Estados miembros sobre la Educación para la Ciudadanía Democrática, de fecha 16 de octubre de 2002, en el que se señala que la educación para la ciudadanía democrática es esencial para promover una sociedad libre, tolerante y justa, y contribuye a defender los valores y principios de la libertad, el pluralismo, los derechos humanos y el imperio de la ley, que son los fundamentos de la democracia. Posteriormente, la Declaración de París de 2015, de los Ministros de Educación de la Unión Europea, ha exigido la adopción de medidas a nivel europeo, nacional, regional y local para reforzar el papel de la educación en la promoción de la ciudadanía y los valores comunes de la libertad, la tolerancia y la no discriminación, subrayando asimismo la necesidad de fortalecer la cohesión social y ayudar a los jóvenes a convertirse en miembros responsables, abiertos y libres de prejuicios, en una sociedad europea diversa e integradora.

No obstante, es la Recomendación 2006/962/CE del Parlamento Europeo y del Consejo de 18 de diciembre, sobre las competencias clave para el aprendizaje permanente, la que define las competencias sociales y cívicas para el desarrollo personal, la ciudadanía activa, la cohesión social y la empleabilidad en una sociedad del conocimiento. Esta recomendación ha tenido una influencia directa sobre los currículos escolares de los países europeos, definiendo conocimientos, habilidades y actitudes para la tolerancia y convivencia democrática y pacífica, para la participación activa y efectiva de los ciudadanos y para el respeto a la diversidad de culturas que conforman el mosaico europeo. De manera expresa este documento hace referencia al necesario aprendizaje de la historia reciente de Europa. La Recomendación de 22 de mayo de 2018 ha actualizado las competencias claves de 2006 y ha recuperado la denominación de competencia ciudadana, incidiendo en la necesidad de un conocimiento y comprensión crítica de los acontecimientos históricos, así como del fomento de la cultura de la paz y la no violencia. 


\section{Los condicionantes curriculares en España.}

La puesta en marcha de la reforma educativa con la LOGSE supuso el inicio de la inclusión de la ciudadanía como contenido curricular en educación secundaria, dentro del octavo bloque de contenidos sobre participación y conflicto político en el mundo actual, aunque en un apartado distinto del referido a España en el mundo y la Comunidad Europea. Posteriormente, en los dos Real Decretos de enseñanzas comunes (uno para la ESO, el otro para el Bachillerato) derivados de la LOCE aparece la expresión "ciudadanía europea". Sin embargo, lo curioso es que aparece hace dentro de las asignaturas de Lenguas Extranjeras, pero no en la de Geografía e Historia o en la asignatura de Ética. Como hemos señalado para el caso de la educación geográfica (De Miguel, 2012) este currículo fue una modificación del desarrollo curricular de la LOGSE y estuvo vigente hasta 2006. Desde entonces, nunca más esta expresión ha figurado en los currículos ministeriales posteriores en desarrollo de la LOE o de la LOMCE, a pesar de que por una investigación de esas fechas ha concluido que existen elementos que deben mejorarse en la enseñanza y el aprendizaje de Europa en los alumnos de la ESO (Prats, 2001).

En 2006 se aprobó la Ley Orgánica de Educación (LOE) que implantó la materia de educación para la ciudadanía y los derechos humanos, como asignatura propia a cursar entre primero y tercero de la ESO, así como la de educación ético-cívica en cuarto y la de filosofía y ciudadanía en primero de Bachillerato. En la norma que estableció las enseñanzas mínimas de la ESO, se hizo expresa referencia a la citada Recomendación del Consejo de Ministros del Consejo de Europa aprobada cuatro años antes. No obstante, el enfoque que se realizaba era excesivamente presentista y enfocado en el mundo actual, y no tenía en cuenta los antecedentes históricos más allá de citar a la Declaración universal de los derechos humanos de 1948, así como una referencia a la valoración de los derechos humanos como conquista histórica inacabada. De hecho, esta asignatura podía ser impartida por profesores de la especialidad de Geografía e Historia como de Filosofía, por lo que en la práctica no hubo un tratamiento didáctico relativo a la vinculación de los procesos históricos que explican la consolidación de las democracias europeas con la adquisición de derechos ciudadanos. 
La Ley Orgánica de Educación fue modificada parcialmente en 2013 por la Ley Orgánica para la mejora de la calidad educativa (LOMCE) que, a pesar de que en su exposición de motivos detalla las implicaciones que tiene para la educación para la ciudadanía las citadas Recomendaciones de 2002 (Consejo de Europa) como la de 2006 (competencias), eliminó las materias relativas a la educación para la ciudadanía y educación ético-cívica e implantó la asignatura de valores éticos, como asignatura alternativa a la religión. De esta manera, la asignatura de Geografía e Historia ha quedado como principal referencia para educar en ciudadanía a los alumnos de la ESO. Por ejemplo, a partir del último criterio de evaluación y estándar de aprendizaje de geografía humana (Bloque 2, del currículo de Geografía e Historia para primer ciclo de la ESO) ${ }^{1}$, losalumnos deben ser capaces de comprender los conflictos bélicos actuales desde factores geopolíticos. En segundo ciclo, hay un contenido curricular expresamente detallado que se refiere al Holocausto (Bloque 6, del currículo de Geografía e Historia para cuarto curso de la ESO), cuyo estándar de aprendizaje plantea a los alumnos que reconozcan la significación del Holocausto en la historia mundial $^{2}$. Igualmente, el bloque octavo trata del mundo reciente entre los siglo XX y $X X I$, y en especial de la transición democrática española y el proceso de construcción de la Unión Europea, como referencias esenciales del fundamento jurídico y político de nuestra ciudadanía española y europea.

Sin embargo, es el bloque décimo el que nos va a permitir desarrollar la siguiente propuesta didáctica al poner en relación el pasado (Holocausto) y el presente (Constitución Española y Tratados de la Unión Europea) con el futuro. Algunas de cuyas tendencias ya empiezan a vislumbrarse en la actualidad en Europa, como los populismos, el incremento de los nacionalismos o las tendencias xenófobas, todo lo cual se aparta de los principios básicos de ciudadanía nacional y europea que hemos detallado anteriormente. Así, el criterio de evaluación referido a que "el pasado no está muerto y enterrado, sino que determina o influye en el presente y en los diferentes posibles futuros y en los distintos espacios" cobra hoy en día especial vigencia ante la incertidumbre política en el escenario geopolítico de enfrentamiento entre Estados

\footnotetext{
${ }^{1}$ Real Decreto 1105/2014.

2 También el Holocausto aparece en el artículo 6 de este mismo Real Decreto como un elemento transversal del currículo para la prevención de cualquier forma de violencia, racismo o xenofobia, así como en el bloque 5 de la asignatura de Historia del Mundo Contemporáneo de $1^{\circ}$ de Bachillerato, y en el bloque 5 de la citada materia Valores Éticos para el primer ciclo de la ESO.
} 
Unidos y China en donde una Unión Europea post-Brexit tiene menos peso como conjunto que alguno de sus principales estados miembros, ante la incertidumbre económica que ha supuesto la crisis derivada del Covid-19 y ante la incertidumbre social del incremento del paro que traerá esta crisis en una estructura demográfica envejecida que sigue necesitando de aportes migratorios que compensen la caída de la natalidad autóctona. En definitiva, a través de la geografía social de Europa en el siglo XXI pretendemos conseguir que los alumnos sean capaces de evaluar cómo una Europa en guerra durante el siglo XX que provocó el Holocausto, ha dado lugar a una unión económica y política cuya integración no debe fragmentarse para garantizar los derechos ciudadanos y la convivencia democrática.

Por último, está en tramitación parlamentaria el proyecto de ley de modificación de la Ley Orgánica de Educación (LOMLOE) que plantea que en un curso de la ESO se imparta de manera obligatoria para todos los alumnos la materia de Valores cívicos y éticos, que incluirá contenidos referidos a los Derechos Humanos y a la Constitución Española, pero también a la educación para el desarrollo sostenible y la ciudadanía mundial, concepto este último incluido dentro de las metas del objetivo 4 de los Objetivos para el Desarrollo Sostenible de Naciones Unidas (ODS).

\section{Algunos recursos didácticos en manuales escolares}

El último bloque de contenidos de cuarto de la ESO ha supuesto un importante avance en el enfoque didáctico de la enseñanza de las ciencias sociales, permitiendo un tratamiento interdisciplinar de la geografía y la historia para la comprensión de la complejidad de las estructuras políticas, económicas y sociales del mundo actual que afronta la tercera década del siglo XXI. Frente al tradicional modelo de los anteriores currículos que solía concluir con temas de España contemporánea, de la Unión Europea y del mundo actual (globalización, conflictos, desequilibrios), el currículo vigente permite comprender mejor las raíces históricas de los procesos actuales y también entender el creciente peso de la geopolítica (De Miguel, 2019) en las relaciones internacionales y en la construcción de la historia del siglo XXI. Hasta el momento, no está caracterizada por grandes conflictos bélicos ni por grandes genocidios como el Holocausto, pero en este siglo las tensiones entre las grandes 
potencias o entre otros poderes menos identificados con un territorio (como las grandes corporaciones o los fondos de inversión transnacionales) no son mucho menores. Pero el devenir del siglo XXI, debido fundamentalmente a la creciente globalización, dependerá cada vez más de la interacción espacial entre unas zonas y otras del planeta en cuestiones como el desarrollo sostenible (véanse las tensiones internacionales derivadas del cumplimiento del Acuerdo de París de 2015 sobre cambio climático), la reorganización espacial de la economía y la sociedad, así como de las innovaciones tecnológicas, algunas de las cuales están todavía por llegar.

Estas cuestiones de evidente actualidad, no siempre están bien resueltas en los manuales escolares de Geografía e Historia para la ESO: los flujos financieros y de información (no siempre visibles ni fáciles de cartografiar), el mundo post-covid19, las tensiones internas en el seno de la Unión Europea y con los países vecinos (Reino Unido, Turquía, Bielorrusia), la estrategia America First o la iniciativa china de la Nueva Ruta de la Seda (Una franja, una ruta). Sin embargo, hay otras aportaciones que merecen ser señaladas como buenos ejemplos de enseñanza de la historia contemporánea desde la geografía a partir de los manuales publicados a partir de 2016 para 4ํ de la ESO en la materia de Geografía e Historia, como posteriormente pretendemos demostrar con los dos estudios de caso analizados.

Por ejemplo, el libro de texto de Vicens Vives recoge dos mapas que tienen una relación directa: el mapa de los conflictos bélicos del siglo XXI y el mapa de las misiones de la ONU para proteger los derechos humanos, especialmente en zonas de África y Oriente Medio, en donde se sigue cometiendo actualmente genocidio, como en Darfur, que sin llegar a las dimensiones del Holocausto, permite establecer una enseñanza comparada de ambos procesos históricos, así como la adquisición de un pensamiento histórico por medio de conceptos como relevancia histórica, causalidad, cambio y continuidad, etc.

El libro de texto de SM desarrolla un taller de simulación del trabajo del historiador a partir del análisis comparativo de los mapas. De esta manera, una de las zonas del planeta en donde se ha dado la mayor violación de los derechos humanos en los últimos años, como es Oriente Medio, y en especial Siria e Iraq, se estudia a través de la cartografía histórica: los Imperios Otomano y Persa en el siglo XVI, el dominio 
británico a principios del siglo XX, la guerra Irán-Irak, la Guerra del Golfo, la Guerra de Irak hasta llegar al Califato del Estado Islámico).

Las dificultades a la convivencia en Europa se recogen en el libro de Anaya por medio del mapa de la Guerra de Yugoslavia o el de los enfrentamientos en el Cáucaso, que precisamente se han reactivado en 2020. Ambos conflictos tienen unos amplios antecedentes históricos y una clara división étnica. En fin, el libro de Santillana permite que alumnos comparen el mapa de Europa en 1988 con el de la Europa actual, para analizar las consecuencias de la desaparición de los regímenes comunistas en Europa oriental, los dos citados conflictos tras la desintegración de la Unión Soviética y de Yugoslavia, así como las dificultades del proceso de ampliación de la Unión Europea hacia el este.

\section{Ciudadanía espacial y educación en valores.}

\section{El proyecto YouthMetre}

El proyecto YouthMetre (Donert et al., 2019) es un proyecto financiado por la Comisión Europea, coordinado por la Asociación Europea de Geógrafos (EUROGEO), conformado por seis socios de otros tantos países europeos, entre ellos el Grupo de Estudios en la Ordenación del Territorio de la Universidad de Zaragoza, que ha diseñado y desarrollado la herramienta principal del proyecto: un visor estadístico y cartográfico on-line que muestra las diferencias espaciales de la población joven en Europa. El acceso al proyecto y a sus resultados se realiza a través de la dirección web http://youthmetre.eu/. Este proyecto se fundamenta en la adquisición de una ciudadanía espacial (Gryl et al., 2010). Para ello, las nuevas tecnologías para la información geográfica, los Sistemas de Información Geográfica (SIG) en línea y los visores cartográficos son imprescindibles en los nuevos estilos de aprendizaje y en la adquisición de competencias espaciales, pero también sociales y cívicas (Gryl y Jekel, 2012). La necesaria integración entre pensamiento espacial y conocimiento geográfico conlleva adicionalmente, el desarrollo de un pensamiento espacial crítico, en definitiva, de una educación en valores que permita a los jóvenes abordar de una forma rigurosa problemas territoriales reales y asumidos por ellos, pero también implicarse a través de la participación ciudadana en buscar respuestas a los mismos, en las ocho 
diferentes dimensiones de la Estrategia de la Unión Europea para la Juventud: Educación y formación; Empleo y emprendimiento; Salud y bienestar; Participación; Actividades de voluntariado; Inclusión social; La juventud en el mundo; Creatividad y cultura.

Un primer mapa es el del índice de desarrollo juvenil (figura 2) que muestra a la escala de las NUT 2 un valor sintético de las ocho dimensiones. Así, al alumno pueden plantearse preguntas del tipo: ¿Existen diferencias territoriales entre unos países de la Unión y otros? ¿Y dentro de cada país? ¿Hay desequilibrios entre el espacio rural y el urbano? De las ocho dimensiones ¿cuál crees que ha podido tener más influencia en el desarrollo social de los jóvenes europeos? Observando la diferencia entre las zonas más altas (valor 98) y más bajas (valor 57 )... ¿crees que vivir en una región europea condiciona las oportunidades de los jóvenes?

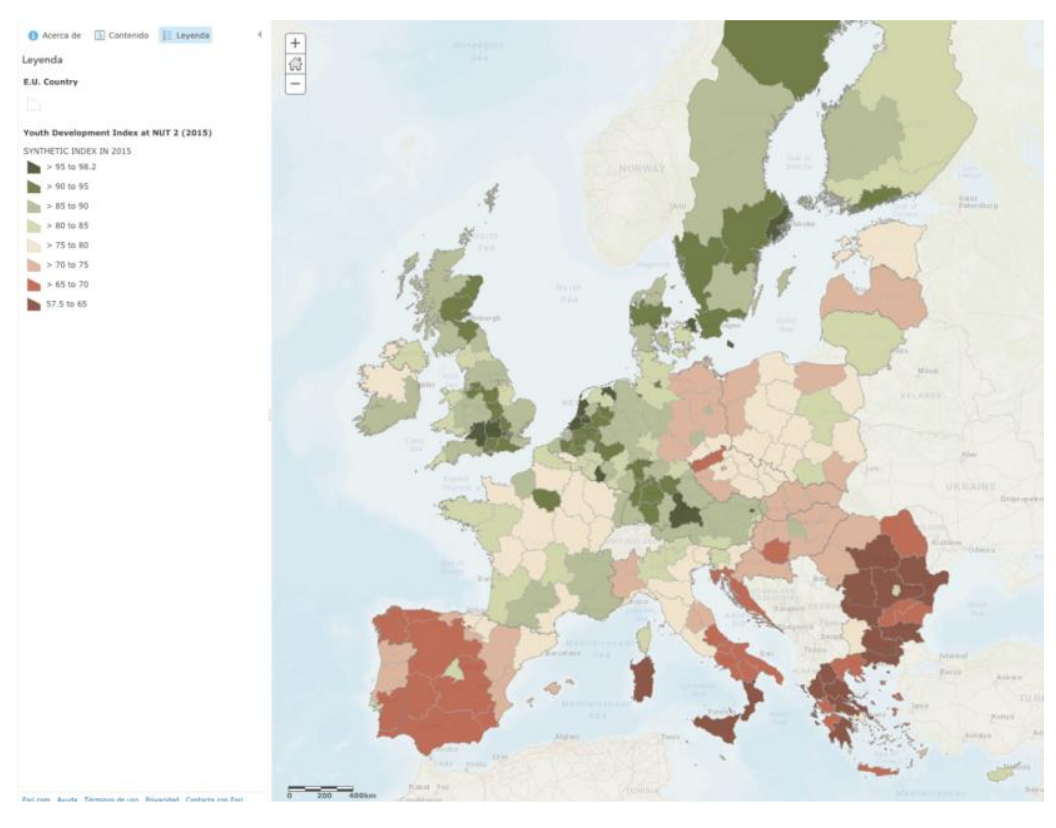

Figura 2. Cartografía sintética (8 dimensiones) del índice de desarrollo de la juventud europea (Proyecto YouthMetre, 2015).

En relación a la enseñanza para la ciudadanía europea, losrecursos aportados por tres de estas dimensiones son especialmente interesantes. La participación de los jóvenes en la vida política -ya sea en elecciones locales, nacionales o europeas- demuestra 
también su nivel de ciudadanía democrática. Entre los mapas de la figura 3 se puede constatar una correlación entre países más abstencionistas con aquellos que en los últimos años ha descendido el interés por ejercer la participación política. Así, podría trabajarse en el aula ambos mapas para que los alumnos identifiquen esos países de manera combinada con alguna de las noticias relacionadas con el Brexit, o con los vetos de Hungría y Polonia a acuerdos del Consejo Europeo, o incluso con las reformas de sus sistemas judiciales, que recientemente han generado suspicacias sobre la separación de poderes y han incrementado el sentimiento nacionalista y euroescéptico.
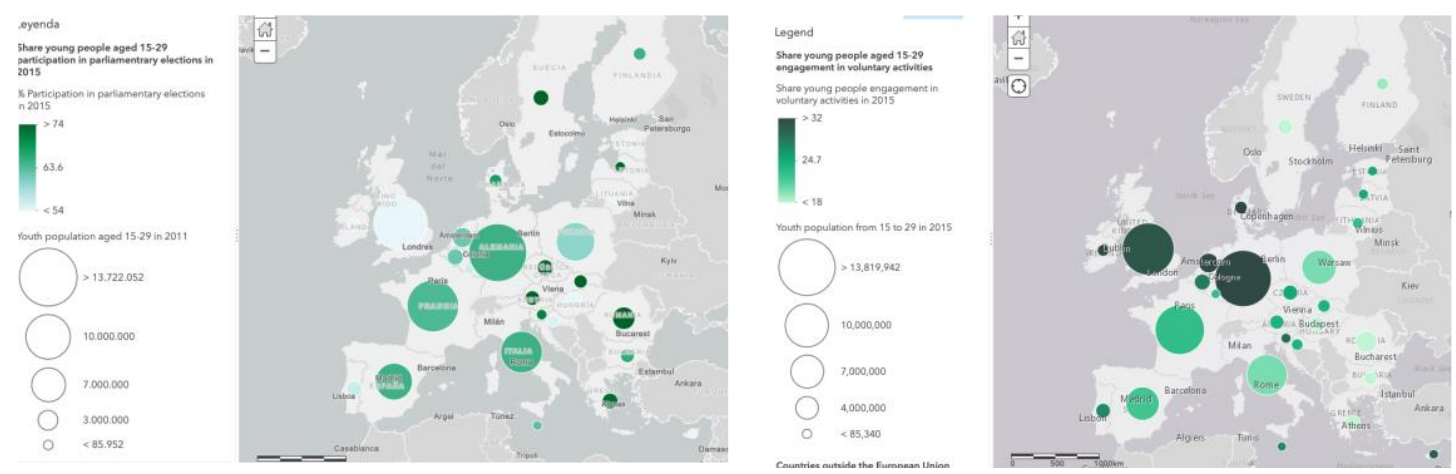

Figura 3. Izquierda: Mapa participación juvenil (15 a 29 años) en las elecciones parlamentarias del 2015. (Proyecto YouthMetre, 2015).Derecha: Mapa participación de los jóvenes europeos (15-29 años) en actividades de voluntariado. (Proyecto YouthMetre, 2015).

Las diferencias existentes entre unos países y otros de la Unión Europea en relación a la implicación de los jóvenes en actividades de voluntariado local (figura 4, mapa de la izquierda) o en participación en ONG relacionadas con derechos humanos (figura 4, mapa de la derecha) también son muy explícitas y permiten visualizar al alumno de la ESO en qué naciones existe una mayor ciudadanía activa o, a la inversa, un mayor rechazo a tendencias antidemocráticas (en cualquiera de los dos extremos de los populismos). También los alumnos pueden comprender, a partir del tema del nazismo y el Holocausto, que hoy en día Alemania es uno de los países europeos en los que sus jóvenes tienen más consolidados los valores ciudadanos, como marcan los valores de los mapas elaborados a partir de las encuestas oficiales de Eurostat. 

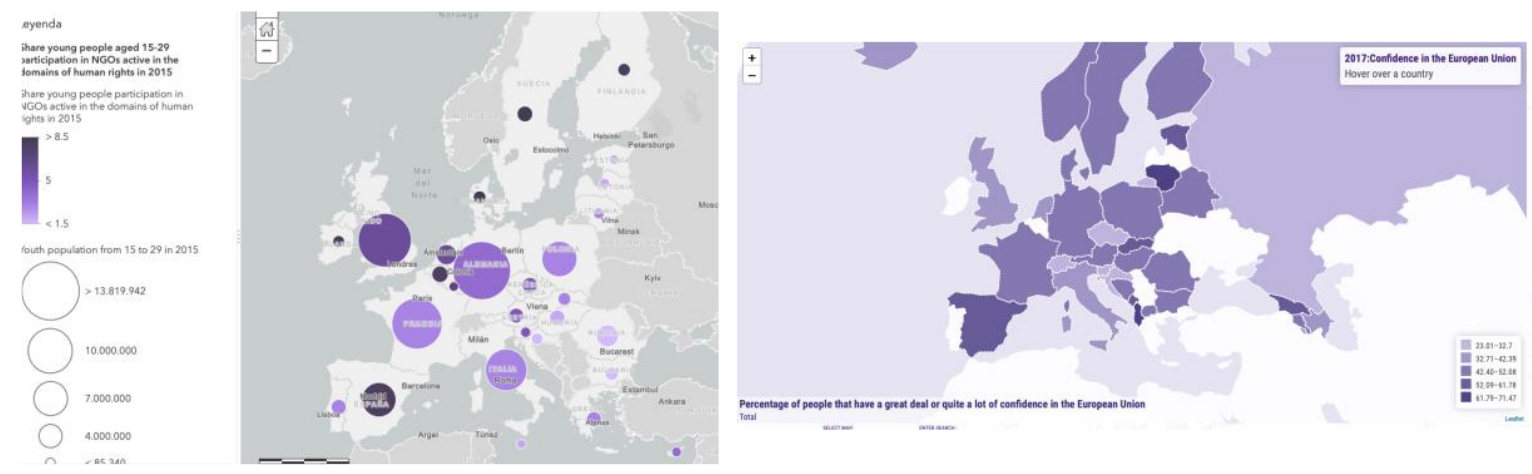

Figura 4. Izquierda: Mapa participación juvenil (15-29 años) en ONGS de derechos humanos (Proyecto YouthMetre, 2015). Derecha: Mapa de confianza de la población en la Unión Europea (Proyecto European Values Study, 2017).

\section{El Estudio de los Valores Europeos}

Otro proyecto en el que EUROGEO también participa es el denominado European Values Study (EVS, en adelante). EVS es un programa de investigación de encuestas longitudinales, transnacionales y a gran escala sobre valores humanos básicos. Proporciona información sobre las ideas, creencias, preferencias, actitudes, valores y opiniones de los ciudadanos de toda Europa. Es un proyecto de investigación único sobre cómo piensan los europeos sobre la vida, la familia, el trabajo, la religión, la política y la sociedad. El Estudio de valores europeos se inició en 1981 y se repite cada nueve años (1981, 1990, 1999, 2008 y 2017). La última ola en 2008 ha cubierto al menos de 35 países europeos y 55.000 personas entrevistadas en toda Europa. Con todos estos datos se ha podido elaborar una cartografía actualizada (puede consultarse en la web https://www.atlasofeuropeanvalues.eu/) sobre los valores de los ciudadanos europeos en más de cien temas diferentes. De todos ellos hemos seleccionado unos pocos, relevantes obre la temática relativa al rechazo a la xenofobia, al totalitarismo, a la violación de derechos humanos y, en consecuencia, a la consolidación de los valores de ciudadanía europea, democracia y convivencia.

La figura 5 muestra el mapa de los países en donde existe un mayor rechazo a tener como vecino a personas de otra raza, que son preferentemente los antiguos países del bloque comunista en Europa central y oriental, con valores superiores al $25 \%$ en varios países frente a las tasas inferiores al $10 \%$ en la mayor parte de los grandes Estados 
de Europa occidental. Investigar los antecedentes históricos de este grupo de países del este puede ayudar a los alumnos a comprender los valores tan elevados. En cierta medida, este mismo patrón espacial se repite ante el mapa (figura 6) que muestra el antisemitismo que existe hoy en Europa. Precisamente aquellos países que tuvieron mayor número de víctimas por el Holocausto (Polonia, Checoslovaquia, Rumanía, Unión Soviética) vienen a coincidir con los territorios en donde actualmente hay una tasa de rechazo a los judíos en torno al $20 \%$ o superior. Por el contrario, en la mayor parte de los países europeos estas cifras apenas alcanzan el 5\%, incluida Alemania (3'9\%), cuyo antecedente histórico del III Reich fue el impulsor del Holocausto. Lo cual no deja de ser una gran lección histórica sobre la que los alumnos de cuarto de la ESO pueden reflexionar.

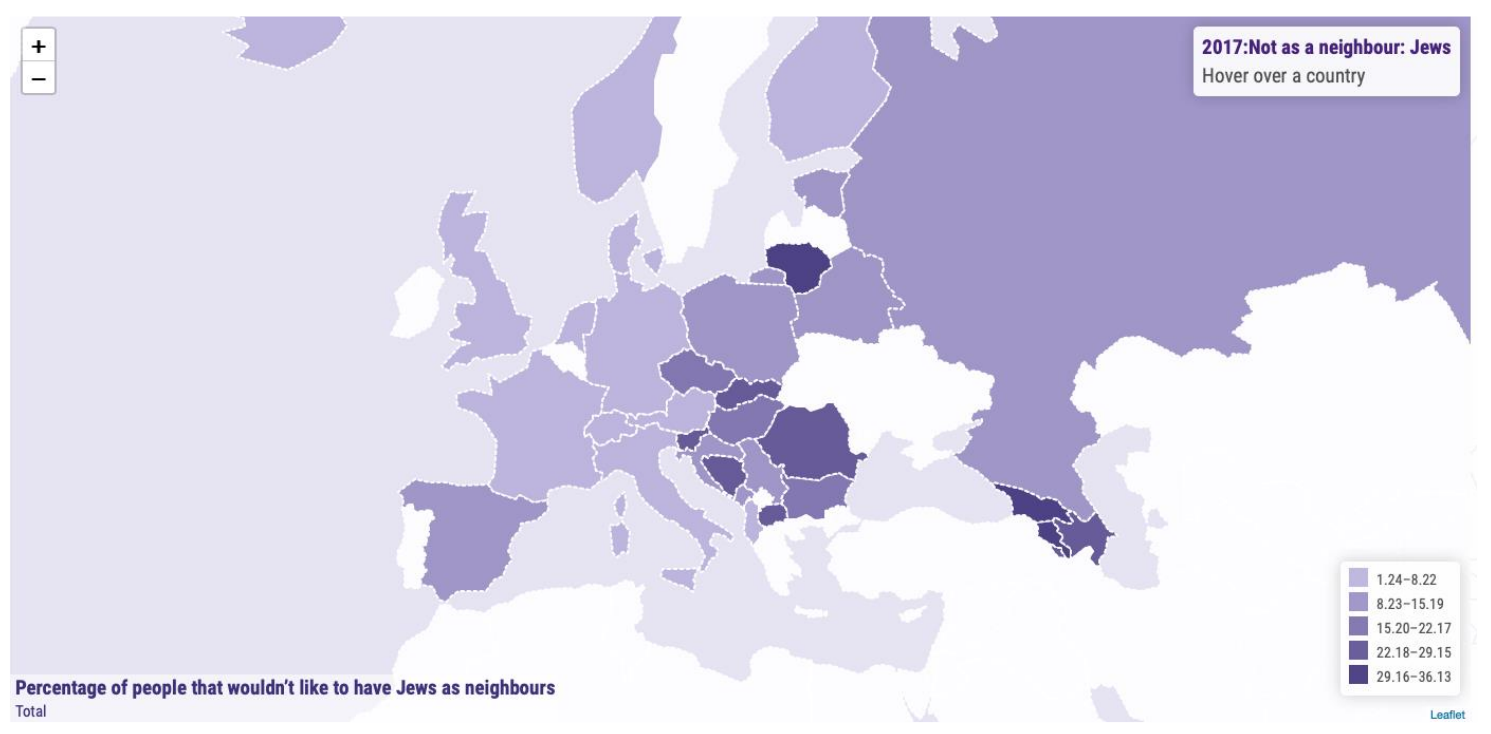

Figura 5: Mapa de rechazo a personas de otra raza (Proyecto European Values Study, 2017). 


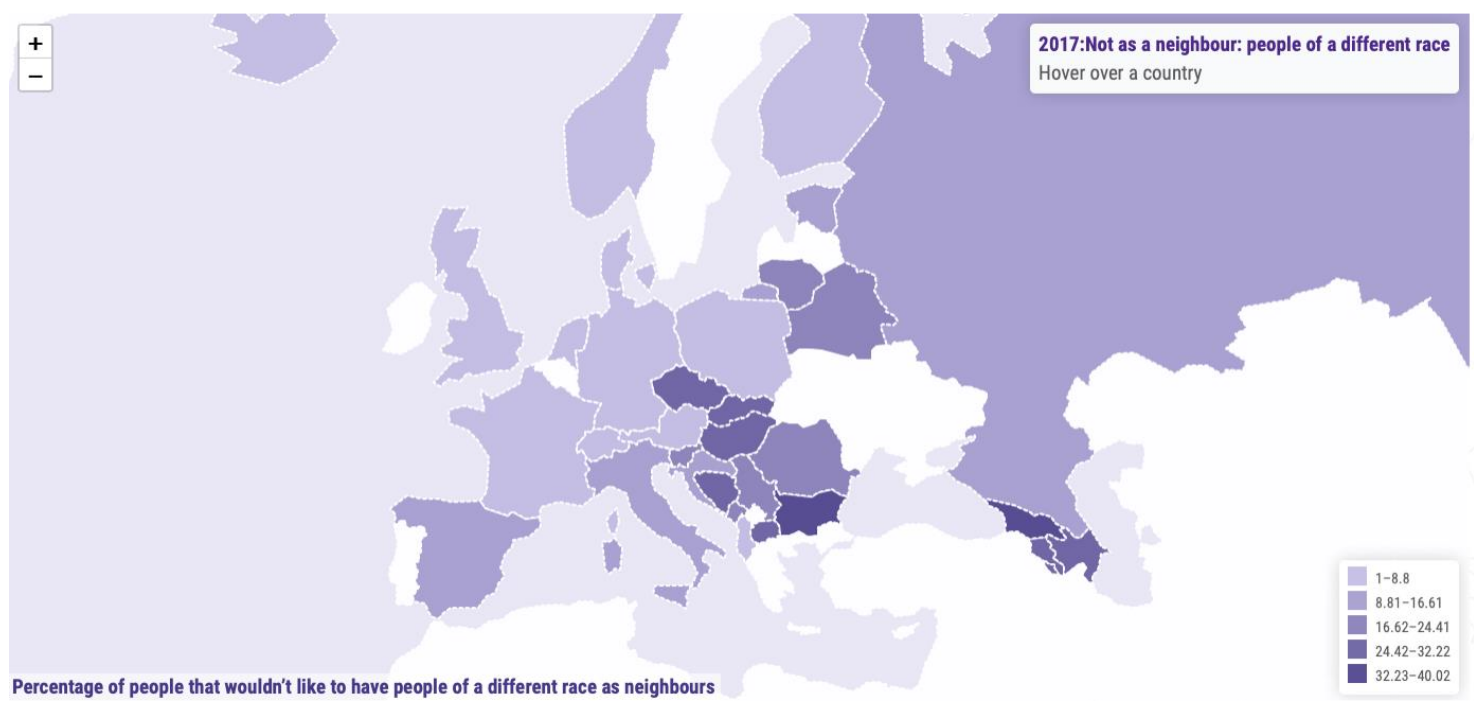

Figura 6: Mapa de rechazo a los judíos (Proyecto European Values Study, 2017).

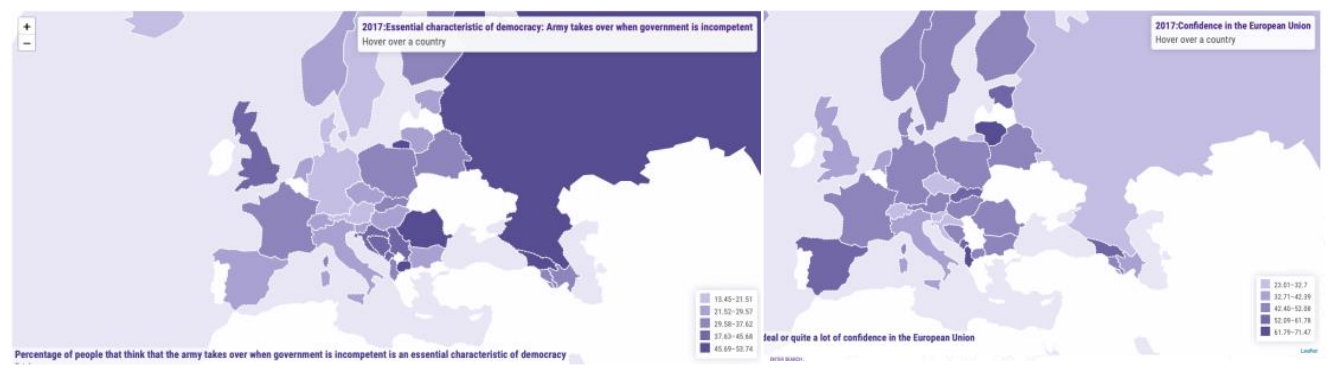

Figura 7: Izquierda: Mapa de valoración de la democracia frente a la intervención del ejército en el gobierno (Proyecto European Values Study, 2017). Derecha: Valoración de la democracia como sistema garantista de los derechos civiles (Proyecto European Values Study, 2017).

Otros cartografías (figuras 7 y 8 ) que sirven para verificar el grado de adquisición de ciudadanía democrática, bien a través del grado de confianza en la Unión Europea, donde España alcanza una de las tasas más altas del continente, del estado de derecho y el imperio de la ley frente la intervención militar (de nuevo el contraste entre Alemania y Rusia es evidente) o la percepción de la democracia como un sistema que protege los derechos individuales frente a la opresión, en donde a pesar de las diferencias entre países se puede constatar que los encuestados de casi todos los países europeos tienen una mayoritaria convicción democrática. 


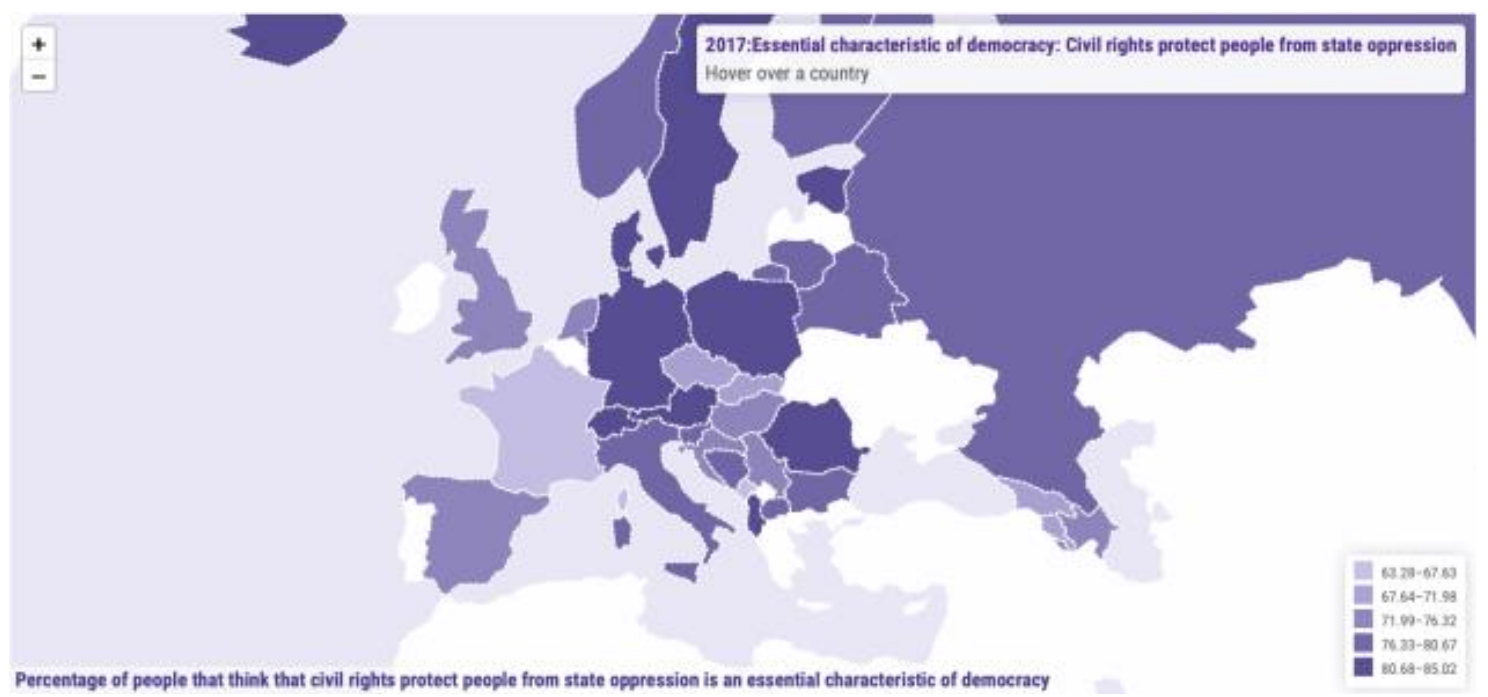

Figura 8: Valoración de la democracia como sistema garantista de los derechos civiles (Proyecto European Values Study, 2017).

\section{Conclusiones}

Tratar el concepto de ciudadanía europea implica abordar temas de derechos humanos, respeto, valores democráticos y libertades esenciales para el ser humano.

En este sentido, es necesario establecer prácticas educativas que desarrollen ese pensamiento critico y democrático y quepromuevan la capacidad de interpretar y evaluar la información a lo largo de la historia.

El uso didáctico de cartografías temáticas a través de plataformas web-SIG, como las presentadas en este artículo, fomenta tanto el análisis geo-histórico de la Unión Europeacomo el pensamiento crítico del devenir histórico de los paises miembros.

Esto implica formar a ciudadanos autónomos, eficientes, responsables, críticos y reflexivos a la hora de seleccionar, tratar y utilizar la información y sus fuentes, así como las diferentes herramientas tecnológicas. Además, conlleva tener una actitud crítica y reflexiva en la valoración de la información disponible, contrastando cuando sea necesario. Por ultimo, promueve la participación de los jóvenes en este sistema democrático y los integra como actores de cambio para la integración social de todos los estados miembros. 


\section{Agradecimientos}

Este trabajo ha sido realizado en el marco del Grupo de investigación ARGOS (Gobierno de Aragón / FEDER, UE) y del Instituto Universitario de Investigación en Ciencias Ambientales de Aragón (IUCA-Universidad de Zaragoza) y EUROGEO.

\section{Referencias}

Caballero, A., Cárdenas, J. F. y Valle, J. M. (2016). La educación para la ciudadanía en la Unión Europea: Perspectivas supranacional y comparada. Journal of supranational policies of education, 5, 173-197.

Comisión Europea / EACEA / Eurydice. (2017). La educación para la ciudadanía en el contexto escolar europeo 2017. Informe de Eurydice. Oficina de Publicaciones de la Unión Europea.

De Miguel. R. (2019). Europe in a global context: EUROGEO and The role of geography and european geographers. European Journal of Geography, 10(4), 160-176.

De Miguel, R. (2012). Análisis comparativo del currículum de Geografía en educación secundaria: revisión y propuestas didácticas. En R. de Miguel, M. L de Lázaro y M. J. Marrón (Eds.), La educación geográfica digital (pp. 13-36). Grupo de Didáctica de la Geografía (A.G.E.) y Universidad de Zaragoza.

Donert, K., De Miguel, R. y Luppi, A. (2019). YouthMetre: Open Data to Empower Young People to Engage in Democracy and Policymaking. En R. de Miguel, K. Donert y K. Koutsopoulos (Eds.), Geospatial technologies in Geography Education (pp. 87-101). Springer.

Gryl, I. y Jekel, T. (2012). Re-centering geoinformation in secondary education: Toward a spatial citizenship approach. Cartographica, 47(1), 18-28.

Gryl, I., Jekel, T. y Donert, K. (2010). GI and spatialcitizenship. En T. Jekel, K. Donert, A. Koller y R. Vogler (Eds.), Learning with GI V (pp. 2-10). Wichmann.

Jara, M. A. y Santisteban, A. (Coord.) (2018). Contribuciones de Joan Pagès al desarrollo de la didáctica de las ciencias sociales, la historia y la geografía en Iberoamérica. Universidad Nacional del Comahue.

Lee, W.O., Grossman, D.L., Kennedy, K.J. y Fairbrother, G.P. (Eds.) (2004). Citizenship Education in Asia and the Pacific. Springer.

Massip, M. y Santisteban, A. (2020). La educación para la ciudadanía democrática en Europa. Rev. Espaço do Currículo, 13(2), 142-152.

Pagés, J. y García, C. R. (2020). Qué piensa y qué escribe Ernesto Gómez. Su aportación a la Educación para la Ciudadanía Democrática. REIDICS, 7, 7-29.

Prats. J. (Dir.) (2001). Los jóvenes ante el reto europeo. Conocimientos y expectativas del alumnado de educación secundaria. Fundación La Caixa. 